\title{
Fibrillary Glomerulonephritis in Primary Sjogren's Syndrome: A Rare Cause of Renal Failure
}

\author{
Rahul Sehgal, MD; Syed M. Saijad, MD; and Jiwan K. Thapa, MD
}

\begin{abstract}
Renal involvement in primary Sjogren's syndrome (PSS) varies in severity and prevalence. Although previously felt to be uncommon, kidneys can be involved in $25 \%$ to $30 \%$ of pSS patients. Fibrillary glomerulonephritis (FGN) is a rare primary glomerular disease that can occur in association with another autoimmune condition or malignancy. The diagnosis relies on renal biopsy findings of haphazardly arranged fibrils in all glomerular compartments and distinction from other forms of fibrillary glomerulopathies such as renal amyloidosis and immunotactoid glomerulopathy. FGN responds poorly to immunosuppressive therapy and has a poor prognosis. Here, we describe a case of FGN in a patient with asymptomatic pSS. We describe the diagnostic work-up, clinical course, treatment utilized, and I-year follow-up. There is one other case in the literature of FGN in a patient with pSS. The rarity of this association and distinction of FGN from other forms of renal involvement in pSS is important as it impacts therapy and prognosis. The case highlights electron microscopy findings in FGN and poor prognosis.
\end{abstract}

Keywords: Fibrillary glomerulonephritis; Sjogren's syndrome; Renal failure; Glomerular disease

$\mathrm{P}$ rimary Sjogren's syndrome (pSS) is a chronic autoimmune disease affecting up to $0.6 \%$ of Americans, with a female predilection and onset in the fourth to sixth decades of life. ${ }^{1,2}$ Sjogren's syndrome is regarded as an autoimmune exocrinopathy characterized by xerostomia and xerophthalmia, which together are referred to as the 'sicca complex'. ${ }^{3}$ Laboratory data reveal increased circulating polyclonal immunoglobulins, rheumatoid factor, antinuclear antibodies, and anti-Ro (SS-A)/anti-La (SS-B) antibodies while focal or diffuse lymphocyte infiltration, predominantly by CD4 T-lymphocytes are key histological features on labial salivary gland biopsy. ${ }^{4} \mathrm{~A}$ variety of non-exocrine glandular involvement including skin, lung, gastrointestinal tract, central and peripheral nervous system, musculoskeletal system, and kidneys may occur in pSS. ${ }^{5,6}$ Renal involvement in pSS may vary in severity and may be minimally symptomatic with electrolyte disturbances, mild proteinuria, and slight renal impairment in tubulointerstitial nephritis or be accompanied with overt nephropathy as seen in membranoproliferative glomerulonephritis. Other disease manifestations including nephrolithiasis and nephrocalcinosis can be seen in distal renal tubular acidosis. These extra glandular manifestations impact patient survival and quality of life.

Fibrillary glomerulonephritis (FGN) is a rare primary glomerular disease presenting with hematuria, renal insufficiency, hypertension and nephrotic range proteinuria. ${ }^{7}$ We present here a case of FGN in a patient with asymptomatic pSS. Currently, there is only one other case of FGN in pSS in the published literature. ${ }^{8}$

\section{Case Presentation}

A Caucasian man, aged 77 years, presented with 6-month history of progressively worsening weakness, weight gain and loss of appetite. His past medical history included hypertension, dyslipidemia and symptomatic nephrolithiasis. His home medications were simvastatin and captopril. He had a 50-pack per year history of smoking which he quit 4 years prior. 


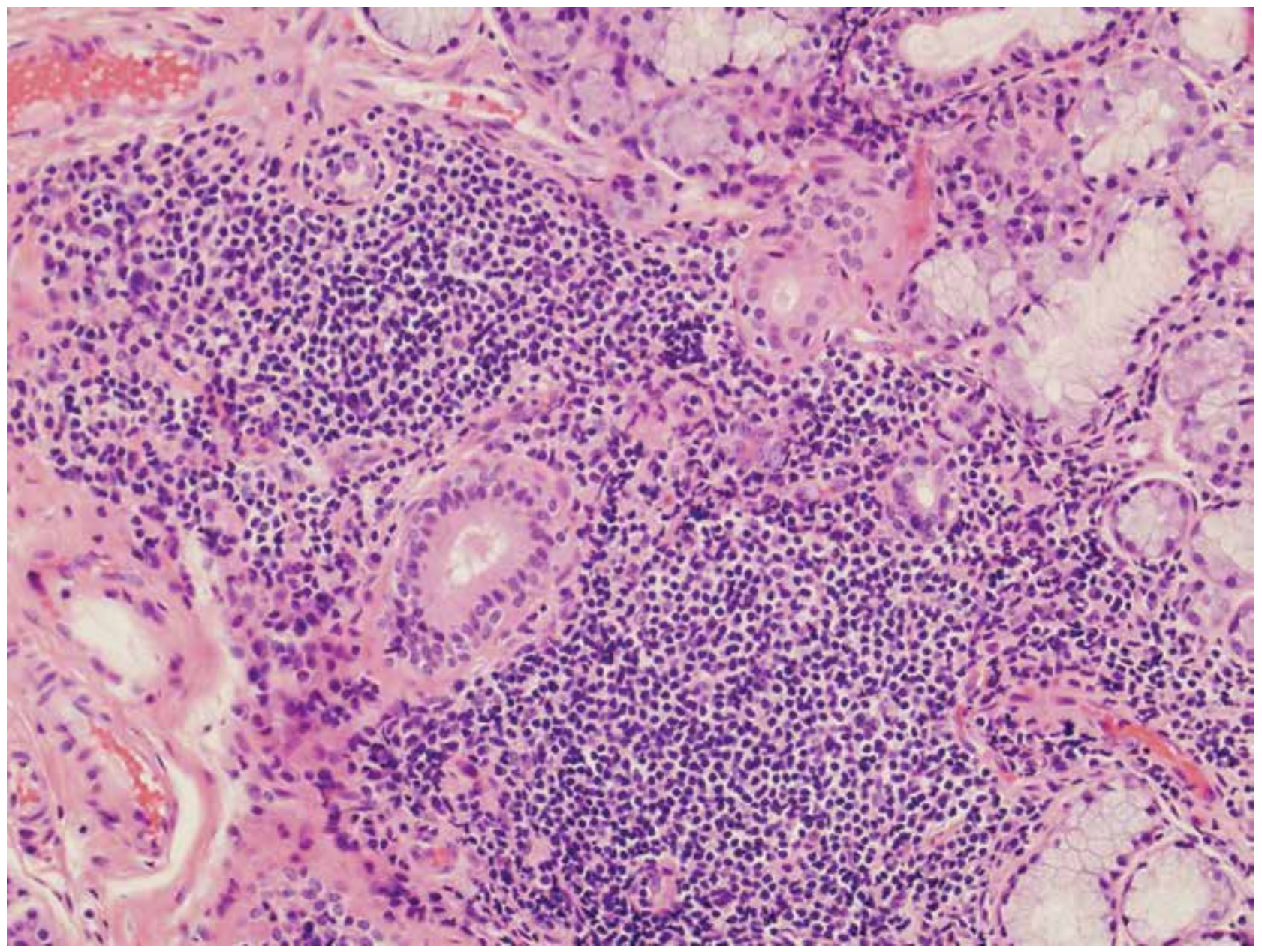

Figure 1. Minor Salivary gland tissue with lymphocytic and plasma cell infiltrate. H\&E stain x200.

On physical examination, the patient was hypertensive with blood pressure of 194/95, heart rate of 60 beats per minute and oxygen saturation of $95 \%$ on room air. He had severe pitting edema in lower extremities and bibasilar rales on chest auscultation. There was no evidence of xerophthalmia or xerostomia and no enlargement of parotid or submandibular glands.

A complete blood count revealed a normal leukocyte count of (5.9 $1,000 / \mu \mathrm{L}$, reference range [RR] 4.1-10.9 $1,000 / \mu \mathrm{L})$, normocytic anemia (hemoglobin 11.6 g/dL, RR 12.9-17.3 g/ $\mathrm{dL}$ ), and normal platelet count. Liver transaminases were normal. Renal function tests revealed elevated creatinine (4.0 $\mathrm{mg} / \mathrm{dL}$, RR 0.6-1.2 mg/dL) and blood urea nitrogen $(70 \mathrm{mg} /$ $\mathrm{dL}$, RR $6-24 \mathrm{mg} / \mathrm{dL}$ ). Urine dipstick revealed evidence of $2+$ blood, and urine spot test showed nephrotic range proteinuria (4.2 g, RR <0.165). Erythrocyte sedimentation rate $(104 \mathrm{~mm} /$ $\mathrm{hr}, \mathrm{RR}<13)$ and C-reactive protein $(20 \mathrm{mg} / \mathrm{dL}, \mathrm{RR}<1.0 \mathrm{mg} /$ dL) levels were elevated. Antinuclear antibody was positive $(1: 640, \mathrm{RR}<1: 80)$ and a polyclonal pattern was seen on serum protein electrophoresis with low albumin $(3.0 \mathrm{~g} / \mathrm{dL}$, RR 4-5.4 $\mathrm{g} / \mathrm{dL}$ ) without a definite monoclonal protein and normal serum free-light chain ratio. Urine immunofixation was negative for monoclonal protein, and tests for hepatitis $\mathrm{B}$ and $\mathrm{C}$ infection were negative as well. Anti-SS-A and anti-SS-B antibodies were positive $(>8, \mathrm{RR}<0.9)$ while antibodies for doublestranded DNA and antiphospholipid antibodies were absent and serum complement levels (C3 and $\mathrm{C} 4)$ were normal. Serum electrolytes and anion gap were normal with metabolic acidosis.

An ultrasound of the urinary tract demonstrated echogenic and atrophic left kidney measuring $8.5 \times 4 \times 4.5 \mathrm{~cm}$ and compensatory hypertrophy of right kidney measuring $13 \times 6.5 \times 7.5 \mathrm{~cm}$. Chest radiography revealed mild cardiomegaly, pulmonary vascular congestion and mild bibasilar pleural effusion. An extensive evaluation for underlying malignancy including computed tomography scan of chest, abdomen and pelvis were negative.

A labial salivary gland biopsy revealed sclerosing chronic sialadenitis with focus score of 8 foci $/ 4 \mathrm{~mm}^{2}$ confirming a diagnosis of Sjogren's syndrome (Figure 1).

In the setting of acute renal failure, nephrotic range proteinuria and hematuria, a right-side renal biopsy was performed. Glomeruli showed diffuse thickening of the capillary basement membranes, increased mesangial matrix and cellularity, 


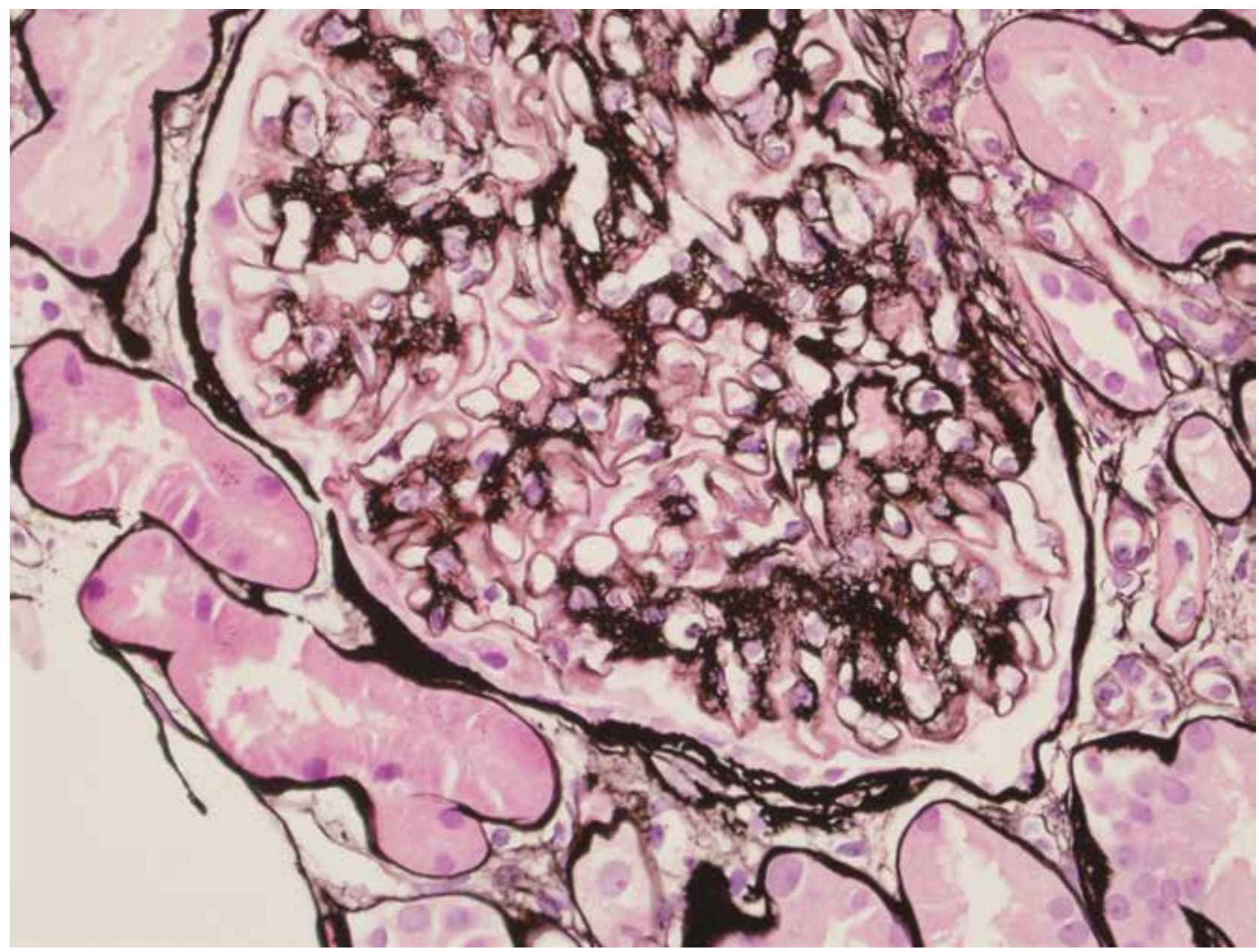

Figure 2. Glomerulus with mesangial expansion \& thickened capillary basement membranes. H\&E stain x400.

without crescents (Figure 2). Mesangial expansion by randomly disposed and non-branching fibrils measuring approximately $12 \mathrm{~nm}$ in diameter was seen under electron microscopy (Figure 3). Extensive involvement of glomerular basement membrane, with accentuation of the outer aspect of basement membrane was seen (Figure 4). Mild interstitial inflammation without tubulitis and marked tubular atrophy and interstial fibrosis and minimal arteriosclerosis of medium and large-sized vessels was seen. Immunofluorescence microscopy showed diffuse segmental to global granular staining along glomerular capillary walls comprising of $\mathrm{IgG}$, C3, lambda, C1q, kappa, IgM, C4d and trace IgA. Congo red stain and liquid chromatography tandem mass spectrometry did not identify a specific amyloid type. The patient's clinical presentation and findings on light microscopy, immunofluorescence and electron microscopy were consistent with a diagnosis of FGN secondary to pSS.

Treatment comprised of oral daily prednisone (40mg), azathioprine and aggressive diuresis. Oral bicarbonate was administered for metabolic acidosis. After 4 weeks, patient continued to have worsening renal failure and hyperkalemia prompting initiation of hemodialysis. He has remained on renal replacement therapy for 1 year and immunosuppressive treatment was discontinued.

\section{Discussion}

The prevalence of renal disease in Sjogren's syndrome ranges from $1 \%$ in retrospective registries ${ }^{9,10}$ to up to $27 \%$ in European studies. ${ }^{11,12,13}$ Renal disease in pSS is usually asymptomatic though overt signs may include hypokalemic paralysis, nephrotic syndrome or recurrent stones complicated by nephrocalcinosis. ${ }^{13}$ Renal disease may sometimes precede onset of sicca symptoms. Although tubulointerstitial nephritis is the most frequent renal complication of pSS, other manifestations including membranoproliferative glomerulonephritis secondary to cryoglobulinemia and distal renal tubular acidosis ${ }^{14}$ may also occur. The prognosis of renal disease in pSS is generally favorable especially in membranoproliferative glomerulonephritis due to early diagnosis while some degree of renal impairment and proteinuria in tubulointerstitial nephritis persists due to delay in diagnosis. ${ }^{14}$ Corticosteroids are considered first line agents in tubulointerstitial nephritis and glomerular disease while data on efficacy of steroid sparing alternatives is lacking. ${ }^{10}$

First reported in 1977 by Rosenmann and Eliakim, ${ }^{7}$ FGN is a rare primary glomerular disease characterized ultrastructurally by glomerular deposition of randomly arranged fibrillary material. ${ }^{15}$ These fibrils resemble amyloid but usually larger in 
diameter and stain negative with Congo red. This is in distinction to a much rarer diagnoses of immunotactoid glomerulopathy characterized by deposition of micro-tubular structures $>30 \mathrm{~nm}$ in diameter. Histologic appearance on light microscopy is heterogeneous and result from varying severity of glomerular capillary wall thickening, mesangial matrix expansion, and hypercellularity, along with membranous glomerulopathy, mesangioproliferative glomerulonephritis, membranoproliferative glomerulonephritis, and crescentic glomerulonephritis. ${ }^{15}$ On immunofluorescence microscopy, the fibrils stain uniformly for immunoglobulins, predominantly IgG4 subclass. ${ }^{15}$

Commonly, FGN presents with hypertension, edema, hematuria and nephrotic range proteinuria. Impaired renal function leading up to renal failure occurs in almost half of affected individuals with FGN. ${ }^{8,15}$ Pathogenesis of FGN is felt to be a result of glomerular localization of immune deposits and subsequent fibrillogenesis. In one of the largest series of 66 cases of FGN reported so far, $75 \%$ of patients had an associated medical condition. This included $40 \%$ patients having an underlying malignancy or autoimmune disease. ${ }^{8}$ Autoimmune diseases associated with FGN from the same study include Crohn's disease, systemic lupus erythematosus, Graves' disease, idiopathic thrombocytopenic purpura, primary biliary cirrhosis, ankylosing spondylitis and one case of Sjogren's syndrome.

Renal involvement can occur in otherwise asymptomatic Sjogren's syndrome patients as was the case presented here. A biopsy should be considered in all cases of suspected renal involvement in pSS to better define underlying disease as it impacts treatment and prognosis. In contrast to a generally favorable prognosis of renal disease in pSS, FGN is poorly responsive to immunosuppressive therapy with most patients developing renal failure within 4 years of their diagnosis. Remission occurs in a small minority of patients and is not affected by therapy utilized.

\section{References}

1. Helmick CG, Felson DT, Lawrence RC, Gabriel S, Hirsch R, Kwoh CK, Liang MH, Kremers HM, Mayes MD, Merkel PA, Pillemer SR, Reveille JD, Stone JH; National Arthritis Data Workgroup. Estimates of the prevalence of arthritis and other rheumatic conditions in the United States. Part I. Arthritis Rheum 2008;58:15-25.

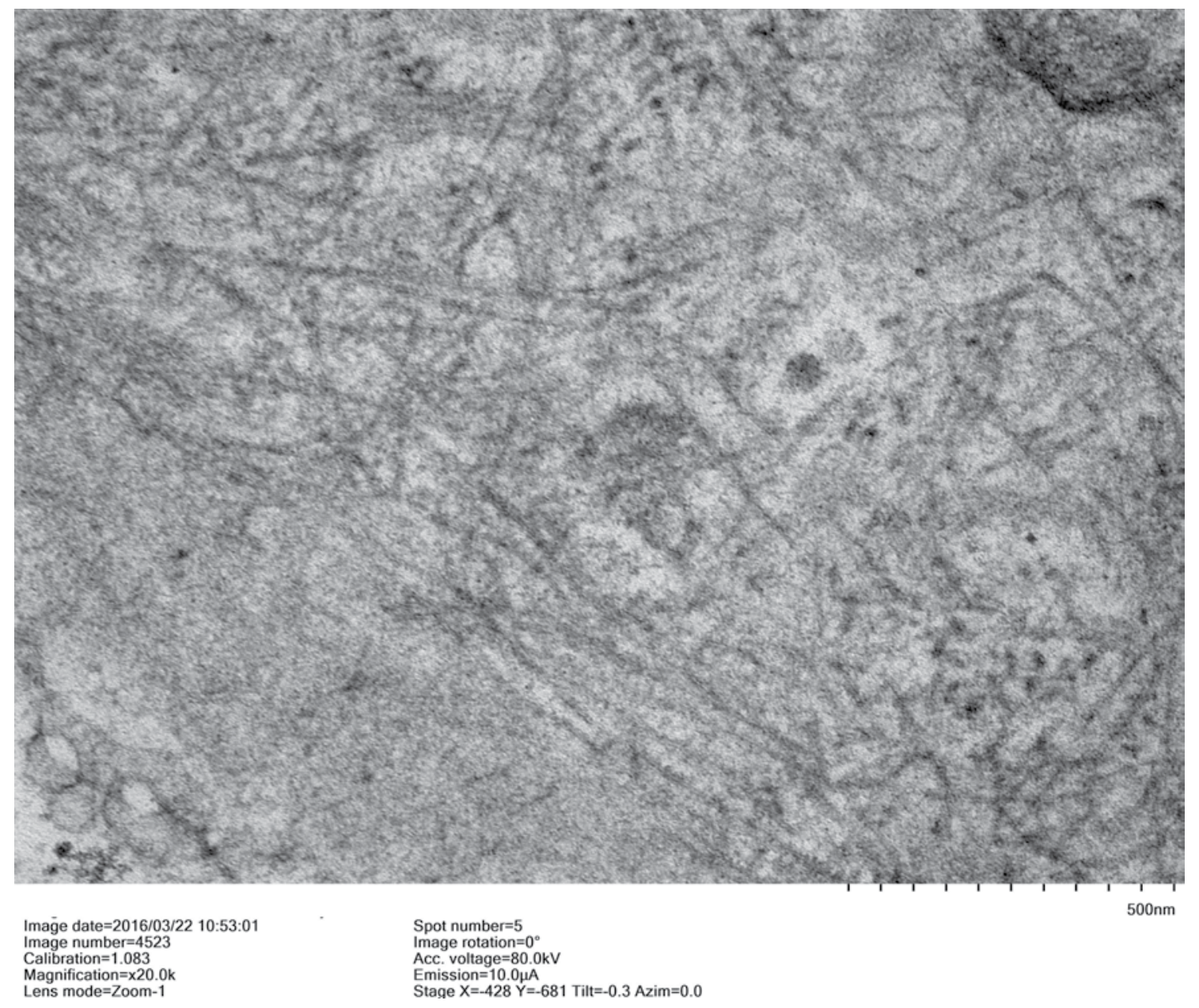

Figure 3. Transmission electron microscopy of glomerular mesangial region with non-branching fibrils which average $12 \mathrm{~nm}$ in diameter. Uranyl acetate \&lead citrate stain x20,000. 
2. Qin B, Wang J, Yang Z, Yang M, Ma N, Huang F, Zhong R. Epidemiology of primary Sjogren's syndrome: a systematic review and meta-analysis. Ann. Rheum Dis 2015;74:19831989.

3. Manoussakis MN, Moutsopoulos HM. Sjogren's Syndrome: current concepts. Adv Intern Med 2001;47:191-217.

4. Alexander EL, Arnett FC, Provost TT, Stevens MB. Sjogren's syndrome: association of anti-Ro (SSA) antibodies with vasculitis, hematologic abnormalities, and serologic hyperreactivity. Ann Intern Med 1983;98:155-159.

5. Bloch KH, Buchanan WW, Wohl MJ, Bunim JJ. Sjogren's syndrome. A clinical, pathological, and serological study of sixty-two cases. Medicine (Baltimore) 1965;44:187-231

6. Manthrope R, Asmussen K, Oxholm P. Primary Sjogren's syndrome: diagnostic criteria, clinical features and disease activity. J Rheumatol Suppl 1997;50:8-11.

7. Rosenmann E, Eliakim M: Nephrotic syndrome associated with amyloid-like glomerular deposits. Nephron 1977;18:301-308

8. Nasr SH, Valeri AM, Cornell LD, Fidler ME, Sethi S, Leung N, Fervenza FC. Fibrillary glomerulonephritis: a report of 66 cases from a single institution. Clin J Am Soc Nephrol 2011;6:775-784.

9. Malladi AS, Sack KE, Shiboski SC, Shiboski CH, Baer AN, Banushree R, Dong Y, Helin P, Kirkham BW, Li M, Sugai S, Umehara H, Vivino FB, Vollenweider CF, Zhang W, Zhao Y, Greenspan JS, Daniels TE, Criswell LA. Primary Sjogren's syndrome as a systemic disease: a study of participants enrolled in an international Sjogren's syndrome registry. Arthritis Care Res (Hoboken) 2012;64:911-918.
10. Maripuri S, Grande JP, Osborn TG, Fervenza FC, Matteson EL, Donadio JV, Hogan MC. Renal involvement in primary Sjogren's syndrome: a clinicopathologic study. Clin J Am Soc Nephrol 2009;4:1423-1431.

11. Seror R, Ravaud P, Bowman SJ, Baron G, Tzioufas A, Theander E, Gottenberg JE, Bootsma H, Mariette X, Vitali C; EULAR Sjogren's Task Force. EULAR Sjogren's syndrome disease activity index: development of a consensus systemic disease activity index for primary Sjogren's syndrome. Ann Rheum Dis 2010;69:1103-1109. Erratum in: Ann Rheum Dis 2011 May; $70: 880$.

12. Goules AV, Tatouli IP, Moutsopoulos HM, Tzioufas AG. Clinically significant renal involvement in primary Sjogren's syndrome: clinical presentation and outcome. Arthritis Rheum 2013;65:2945-2953.

13. Bossini N, Savoldi S, Franceschini F, Mombelloni S, Baronio M, Cavazzana I, Viola BF, Valzorio B, Mazzucchelli C, Cattaneo R, Scolari F, Maiorca R. Clinical and morphological features of kidney involvement in primary Sjogren's syndrome. Nephrol Dial Transplant 2001;16:2328-2336.

14. Francois H, Mariette X. Renal involvement in primary Sjogren's syndrome. Nat Rev Nephrol 2016;12:82-93.

15. Iskandar SS, Falk RJ, Jennette JC. Clinical and pathologic features of fibrillary glomerulonephritis. Kidney Int 1992;42:1401-1407.

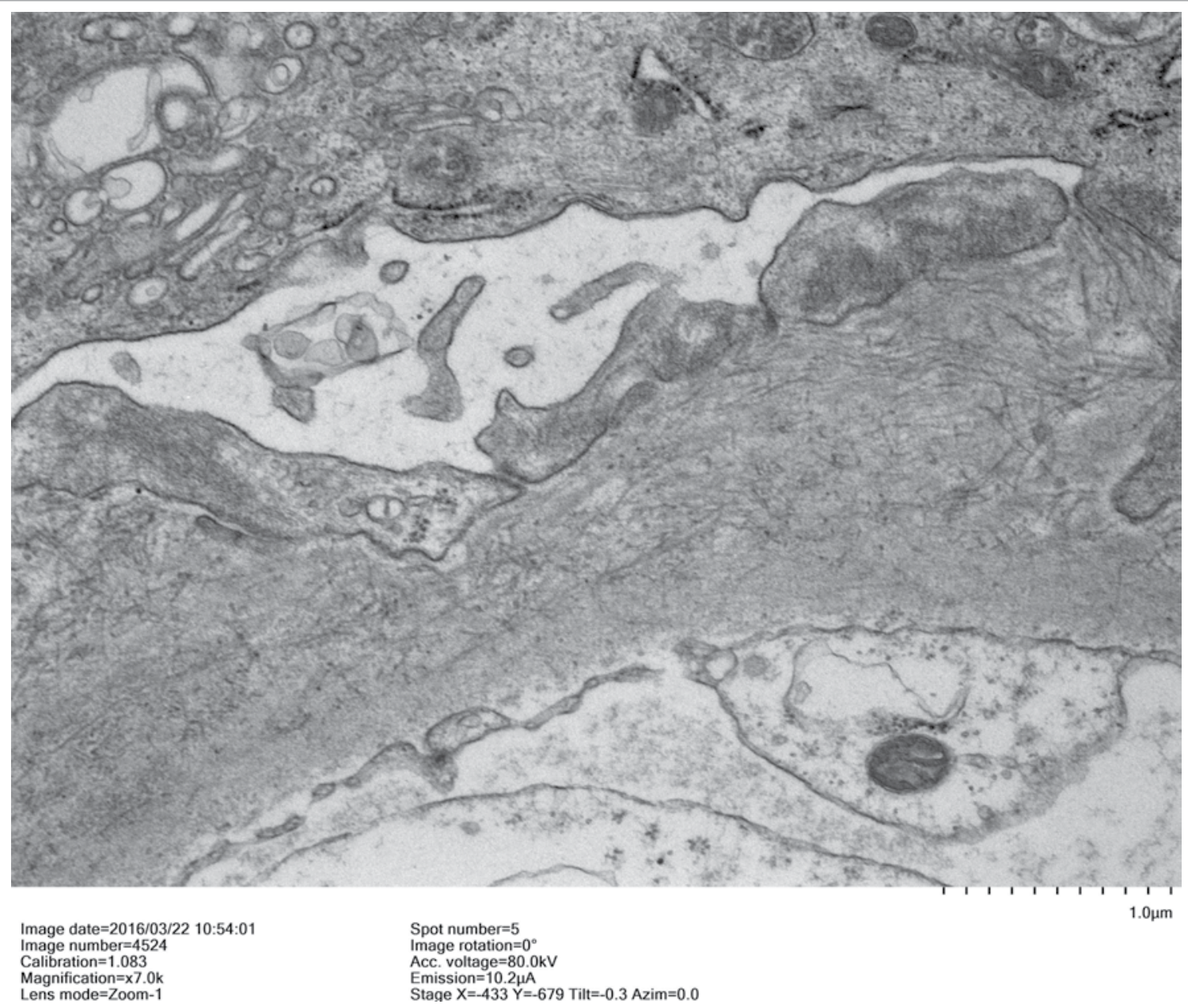

Figure 4. Transmission electron microscopy of glomerular basement membrane with randomly disposed fibrils. Uranyl acetate \& lead citrate stain $\times 7000$. 


\section{Author Affiliations}

Rahul Sehgal, MD*; Syed M. Sajjad, MD广; and Jiwan K.

Thapa, $M D \ddagger$

*Department of Rheumatology, Mayo Clinic, Eau Claire,

Wisconsin, USA

†Department of Pathology, Marshfield Clinic Health System,

Marshfield, Wisconsin, USA

$\neq$ Department of Nephrology, University of Minnesota,

Minnesota, USA 